\title{
Exploration of vascular adhesion protein-1 expression in patients with conjunctivitis associated systemic lupus erythematosus using 2D-DIGE
}

\author{
HONGWEI LIU ${ }^{1 *}$, JIAN ZHANG ${ }^{*}$, PINGPING ZHOU ${ }^{1}$, HAO SUN $^{2}$, \\ MARTHA KATSAROU ${ }^{3}$ and NIKOLAOS DRAKOULIS ${ }^{3}$
}

\begin{abstract}
${ }^{1}$ Department of Ophthalmology, The First Affiliated Hospital of Jiamusi University, Jiamusi, Heilongjiang 154003;
${ }^{2}$ Department of Ophthalmology, The Huai'an Affiliated Hospital of Xuzhou Medical University, Huai'an, Jiangsu 223002, P.R. China; ${ }^{3}$ Research Group of Clinical Pharmacology and Pharmacogenomics, Faculty of Pharmacy, School of Health Sciences, National and Kapodistrian University of Athens, 15771 Athens, Greece
\end{abstract}

Received October 8, 2018; Accepted March 14, 2019

DOI: $10.3892 /$ etm.2019.8009

\begin{abstract}
Conjunctivitis associated systemic lupus erythematosus (caSLE) is a connective tissue autoimmune disease with a large spectrum of clinical manifestations. The disease may lead to ocular complications and in severe cases may be sight-threatening. This study investigated the expression of vascular adhesion protein-1 (VAP-1) in SLE patients with conjunctivitis and the relationship between VAP-1 and other proteins in the onset of the disease. Ten patients with caSLE (caSLE group) and 10 healthy volunteers (control group) were enrolled in the study, in order to evaluate the VAP-1 expression levels in blood. Protein expression profiling was performed with two-dimensional fluorescence difference gel electrophoresis (2D-DIGE) and analyzed with matrix-assisted laser desorption/deionization time-of-flight (MALDI-TOF) used in tandem with mass spectrometry. In the caSLE group, 8 proteins were expressed differenty compared with the control group: C-reactive protein, hemoglobin subunit $\beta$, VAP-1, A-albumin (AFAM), enolase and immunoglobulin heavy
\end{abstract}

Correspondence to: Dr Hao Sun, Department of Ophthalmology, The Huai'an Affiliated Hospital of Xuzhou Medical University, 62 Huaihai South Road, Huai'an, Jiangsu 223002, P.R. China

E-mail: sunhaoshsh111@163.com

*Contributed equally

Abbreviations: VAP-1, vascular adhesion protein-1; caSLE, conjunctivitis associated systemic lupus erythematosus; 2D-DIGE, two-dimensional fluorescence difference gel electrophoresis; AOC3, copper containing 3; CRP, C-reactive protein; HBB, hemoglobin subunit $\beta$; AFAM, A-albumin; ENOA, enolase; IGHM, immunoglobulin heavy constant $\mathrm{mu}$; IRF-1, interferon regulatory factor-1; SAA2, serum amyloid A2 protein

Key words: systemic lupus erythematosus, proteomics, VAP-1, conjunctivitis, VAP-1 protein constant mu were upregulated; interferon regulatory factor-1 and serum amyloid A2 protein were downregulated. Western blotting results are consistent with the proteomics results, showing that in caSLE group VAP-1 expression is increased in comparison to the control group. VAP-1 protein participates in the inflammatory reaction in the form of amine oxidase, copper containing 3. Bioinformatics analysis suggested that VAP-1 protein could be used as a candidate protein for further study regarding its role as a potential protein marker for screening and effectively monitoring caSLE.

\section{Introduction}

Conjunctivitis associated systemic lupus erythematosus (caSLE) is an autoimmune disease of connective tissue with a large spectrum of clinical manifestations (1). Immune deregulation leads to overproduction of autoantibody and immune complexes, complement activation, and persistent tissue inflammation. This may lead to uveitis, fundus changes or optic neuropathy. In severe forms it may lead to sudden loss of vision; the exact mechanism not being fully elucidated yet. To date, several SLE disease-causing antibodies (ABs) have been identified, including anti-double-strand DNA ABs, anti-nuclear ABs and anti-clq antibodies. These ABs have been associated with SLE and lupus nephritis activity. In clinical practice Abs are currently widely used (2-5), while caSLE research remains challenging.

Novel molecular genetic and genomic tools, proteomics and transcriptomics are also used in order to study in depth various diseases.

Vascular adhesion protein-1 (VAP-1) is an endothelial adhesion molecule found in patients with synovitis $(6,7)$, being associated with white blood cells (granulocytes) extravasation from blood into inflammatory tissue. In the muscular stratum of the blood vessels and other tissues, VAP-1 is either tissue-bound or is found in soluble isoforms. The tissue-bound form seems to play a role in cellular differentiation, lipid trafficking and deposition of extracellular matrix components in the smooth muscle $(8,9)$. The soluble isoform is both a 
pre-inflammatory protein as well as an adhesion molecule, a primary AOC3 amine oxidase and a regulator for leucocyte recruitment. This form plays a role in the clinical manifestation of the disease (9).

The aim of this study was to investigate the expression of VAP-1 in caSLE patients and to study the relationship between VAP-1 and other proteins in the onset of the disease.

\section{Materials and methods}

Sample collection. In the present study, 10 patients with caSLE (caSLE group) and 10 healthy individuals (control group) were recruited. The caSLE group were treated in the Jiamusi University, the First Affiliated Hospital (Heilongjiang, China) between February 2015 and February 2017. The study was approved by the Ethics Committee of the First Affiliated Hospital of Jiamusi University (Heilongjiang, China). All the patients signed an informed consent to participate in the study.

Sample preparation. Blood serum samples were collected from each volunteer. Depletion of albumin/IgG followed, using ProteoExtract Albumin/IgG Removal kit (EMD Biosciences) according to the manufacturer's instructions. All samples were concentrated and desalted by ultrafiltration using a 3-kDa cutoff Centrifugal Filter Device (EMD Millipore). 2-D Quant kit (GE Healthcare) was used for determination of protein concentration. Depleted sera were stored at $-80^{\circ} \mathrm{C}$ for further analysis. Three CyDye DIGE fluors (GE Healthcare), Cy2, Cy3 and Cy5 protocol previously described by Hotte and Deyholos (10) was used for labelling protein extracts.

Two-dimensional gel electrophoresis image analysis. The method used for 2-D electrophoresis was previously described by Liu et al (11). The reagents and equipment used for first dimension were immobilized pH gradient (IPG) strips $(24 \mathrm{~cm}$; pH 4.0-7.0); Ettan IPGphor 3 isoelectric focusing system; and SDS-PAGE gels prepared on a low fluorescence borosilicate glass plate (all from GE Healthcare). For the second dimension separation Ettan DALT six electrophoresis system, and IPG strips (24 cm; pH 4.0-7.0) (both from GE Healthcare), Coomassie brilliant blue G-250 (Bio-Rad Laboratories, Inc.) were used. All images were collected on a Typhoon Trio Variable Mode Imager (GE Healthcare). After the protein spot analysis those with significant differences in abundance ( $>1.5$-fold) were selected for spot picking by mass spectrometry.

MALDI mass spectrometer. The protein spots were measured using a 4800 MALDI-TOF/TOFTM Analyzer (Applied Biosystems). The method was previously described by Junker et al (12). GPS Explorer software (version 3.6; Applied Biosystems) was used for combined database search of MS and MS/MS measurements. Peak lists were compared with the SwissProt database v56.1 human taxonomy was used for comparison of peak lists.

Enzyme-linked immunosorbent assay (ELISA). The expression of identified serum levels of VAP-1 proteins was confirmed using ELISA method as previously described by Junker et al (12). ELISA kit (no. 20201ES90; Micro BCA

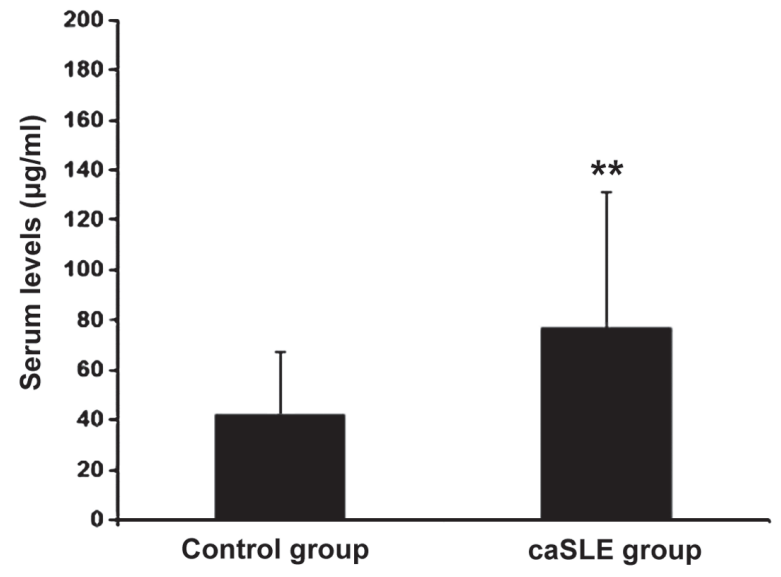

Figure 1. ELISA assay results for VAP-1 levels in control group and caSLE patients group. Values are expressed as mean $\pm \mathrm{SD}$. ${ }^{* *} \mathrm{P}<0.01$.

Protein Assay kit; Thermo Fisher Scientific, Inc.) for VAP-1 was used according to the manufacturer's specifications.

Western blotting. Western blotting was performed according to the methodology of Lappas (13). RIPA buffer was used (100 $\mu \mathrm{l}$; Gibco; Thermo Fisher Scientific, Inc.). BCA Protein Assay (Pierce) was used to determine the protein concentration and $20 \mu \mathrm{g}$ of protein/lane was separated via SDS-PAGE on a $12 \%$ gel. The separated proteins were subsequently transferred onto a polyvinylidene difluoride membrane (EMD Millipore) and blocked for $1.5-2 \mathrm{~h}$ at $34^{\circ} \mathrm{C}$ with $1 \%$ bovine serum albumin (BSA). Following antibodies were utilized: primary antibodies, RhoB (dilution 1:1,000; cat. no. ab53743, Abcam), and $\beta$-actin (dilution 1:20,000, cat. no. ab8227, Abcam), secondary antibodies conjugated with horseradish peroxidase (HRP) (dilution 1:10,000, cat. no. ENZ-321; GE Healthcare). Protein bands were visualized using the Pierce ${ }^{\mathrm{TM}}$ ECL Plus Western Blotting Substrate (Pierce; Thermo Fisher Scientific, Inc.). Quantity One 4.2.1 image analysis software (Bio-Rad Laboratories, Inc.) was used for quantitative analysis of the bands obtained in western blot analyses.

Statistical analysis. All the samples were worked in triplicates. Numerical continuous variables were expressed as the mean \pm the standard deviation (mean \pm SD). Data were analysed using Stata software (Stata 15.1; StataCorp). Comparison between the two groups was performed by Student's t-test. The bioinformatic analysis was performed using STRING database (version 8.3; http://string.embl.de/) in order to identify the proteins for further study.

\section{Results}

Validation of altered VAP-1 by ELISA. The caSLE group VAP-1 levels were increased as compared to the control group, (risk-ratio $=2.77 ; \mathrm{P}<0.01)$ (Fig. 1).

Electrophoresis of fluorescein markers. In Fig. 2 the distribution of protein expression assessed by immune fluorescence in patients with caSLE and conjunctivitis is presented. Among the caSLE patient samples and controls we identified different 
Table I. Differential protein.

\begin{tabular}{lllrr}
\hline $\begin{array}{l}\text { Uniprot } \\
\text { accession no. }\end{array}$ & \multicolumn{1}{c}{ Name } & \multicolumn{1}{c}{ Symbol } & Average ratio & $\begin{array}{r}\text { P-value } \\
\text { t-test }\end{array}$ \\
\hline P02741 & CRP_HUMAN C-reactive protein & CRP_HUMAN & +2.79 & $<0.001$ \\
P68871 & Hemoglobin subunit $\beta$ ( $\beta$-globin) HBB & HBB_HUMAN & +2.34 & $<0.001$ \\
Q16853 & Vascular adhesion protein-1, VAP-1 & VAP-1_HUMAN & +11.72 & $<0.001$ \\
P43652 & Afamin (A-albumin, A-Alb) AFM & AFAM HUMAN & +2.02 & $<0.001$ \\
P06733 & Plasminogen-binding protein, ENOA & ENOA_HUMAN & +1.79 & $<0.001$ \\
P01871 & Immunoglobulin heavy constant mu, IGHM & IGHM_HUMAN & +2.54 & $<0.001$ \\
P10914 & Interferon regulatory factor-1, IRF-1 & IRF1_HUMAN & -1.71 & $<0.001$ \\
P0DJI9 & Serum amyloid A-2 protein, SAA2 & SAA2_HUMAN & -1.73 & $<0.001$ \\
\hline
\end{tabular}

A

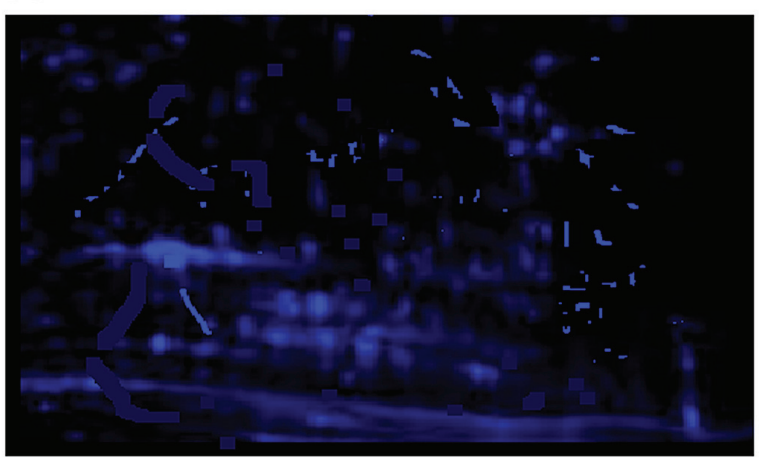

C

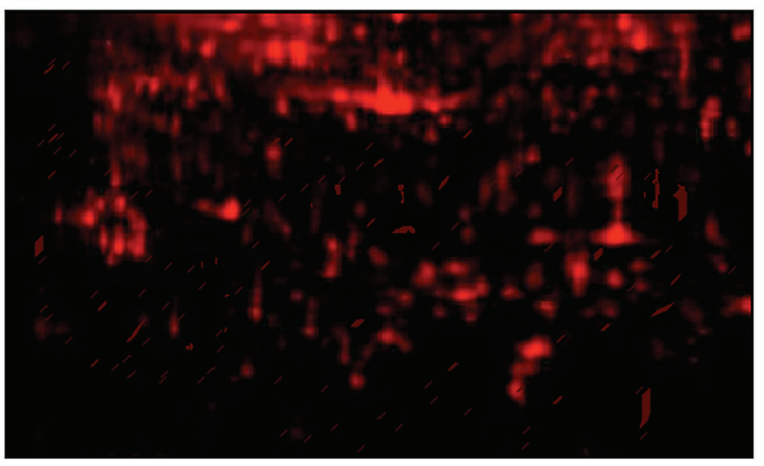

B

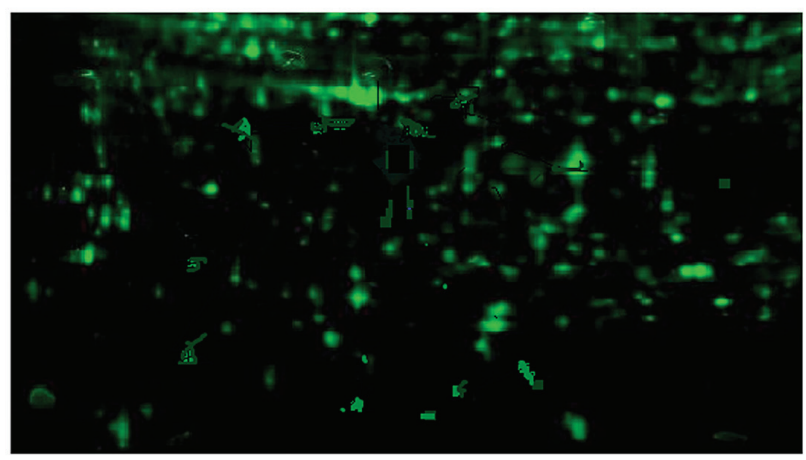

D

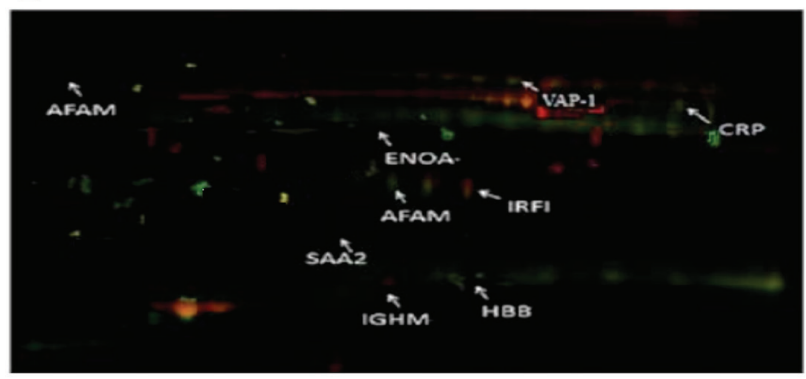

Figure 2. Distribution spots of differentially expressed proteins from blood samples among patients with caSLE and controls in fluorescence difference gel electrophoresis (DIGE). Blood samples from patients with caSLE were labelled with Cy3 dye while Cy5 was used to label the control samples. Cy2: Marks internal standard samples. Differentially expressed proteins identified in 2-DE gels. (A) Labeled with Cy2; (B) Labeled with Cy3; (C) Labeled with Cy5; (D) Merged Cy3 and Cy5. Magnification, x100.

expression in 8 proteins, 2 of which were downregulated while 6 were upregulated (Table I and Fig. 2).

Mass spectrometry analysis, revealed six upregulated proteins including $\mathrm{C}$-reactive protein (CRP), hemoglobin subunit $\beta$ (HBB), VAP-1, A-albumin (AFAM), enolase (ENOA), immunoglobulin heavy constant mu (IGHM), and two downregulated proteins: interferon regulatory factor-1 (IRF-1) and serum amyloid A2 protein (SAA2) (Fig. 2 and Table I).

Bioinformatics analysis. Using an online tool for identifying and analyzing the differentially expressed proteins Metacore software (GeneGo Inc.) it was demonstrated that these differences were involved in various biological processes of protein such as growth, secretion, pathogenesis, translation, cell apoptosis, death, digestion and signal transduction (Table I). Interestingly, it was shown that the VAP-1 protein participates in thirteen important biological processes.

Western blotting. Plasma proteins were extracted from the caSLE and the control group respectively. VAP-1 protein expressed in the control group has a significantly lower expression level than the caSLE patient group, which was 


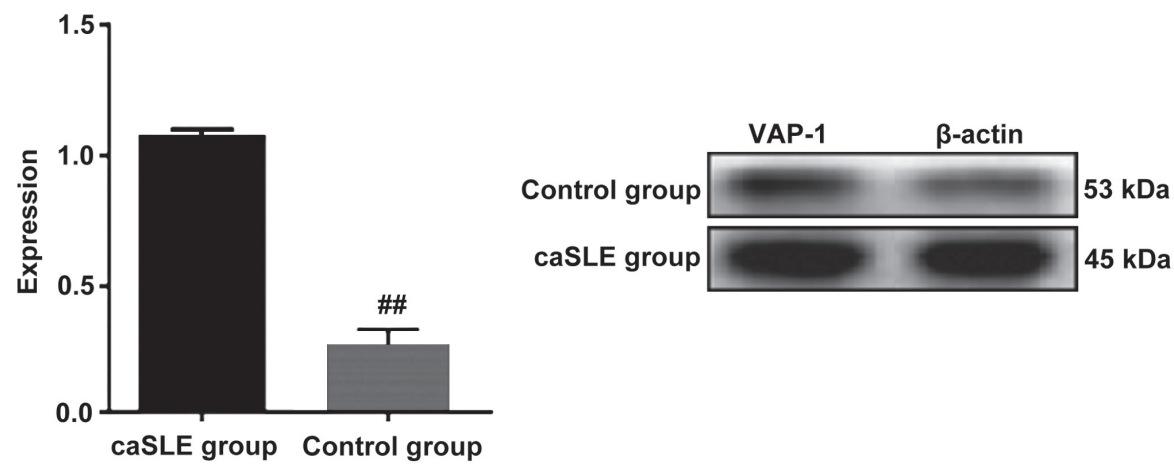

Figure 3. Expression of VAP-1 proteins analyzed by western blotting. Values are expressed as mean $\pm \mathrm{SD}$. ${ }^{\sharp \#} \mathrm{P}<0.01$.

A

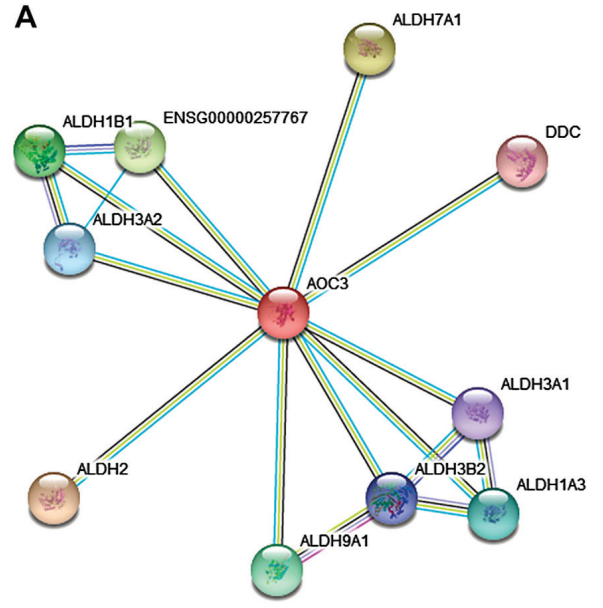

B

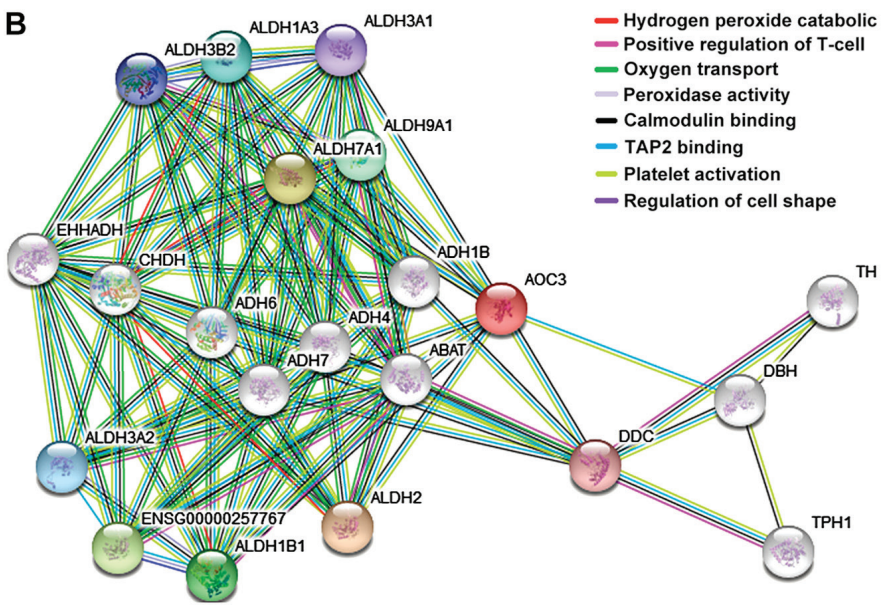

Figure 4. (A) Comparison of protein network expression of eight platelet proteins differentially expressed between healthy control and test groups using Metacore software; (B) STRING visualization of VAP-1 related proteins.

consistent with the results of two-dimensional electrophoresis. A significant difference between the two groups was observed (Fig. 3).

Expression of VAP-1 in database. We used String database to search protein VAP-1. According to the network structure, both control and caSLE groups involved multiple biological processes; positive regulation of T-cell mediated (12.41\%); oxygen transport (5.21\%); peroxidase activity binding (28.71\%); and catalytic activity (18.68\%) (Fig. 4).

\section{Discussion}

SLE is a common autoimmune disease. Promotion of inflammatory cytokines and anti-inflammatory cytokines in humans play an important role in the occurrence and development of SLE as well as in the disease associated multiple organs involvement $(8,14)$. This comparative proteomics study found a number of proteins that are differentially expressed in caSLE affected individuals.

The wide application of proteomic techniques has led to many studies in the field of caSLE (8). Some results in this study were achieved using proteomics, however application of proteomics in the present study was methodologically different.

Proteomics research based on fluorescence differential bidirectional gel electrophoresis (2-DIGE) is a new trend.
2-DIGE has many advantages over conventional 2-DE: The use of 3 different fluorescent dyes for marking can separate multiple samples with different fluorescent tags on the same glue. Different fluorescence labeling samples have different excitation wavelengths, which can record the results of each non-interference with different filters. Avoiding the error of glue, to reflect the real degree of protein change, thus improving accuracy; using an internal standard, the application software can automatically calibrate expression according to the internal standard of each protein point; reduce the error; there are fewer human factors to ensure higher throughput and experimental accuracy $(12,15-19)$. In a mouse model study with late-onset systemic lupus erythematosus E2F2, Azkargorta et al (20) showed using two-dimensional fluorescence difference gel electrophoresis (2D-DIGE) and mass spectroscopy, that when comparing T-lymphocytes in the study group and in the control group, a significant difference in T-cells and protein expression was implicated in receptor mediated stress response, signal transduction and cell survival.

This study used coremine database (http://www.coremine. $\mathrm{com} /$ ) to identify differentially expressed proteins in 16 different proteins. These proteins are involved in 1,446 different biological processes, former eight biological processes, growth, secretion, pathogenesis, translation, cell apoptosis, death, digestive and signal transduction. The newly found biological processes include cell stimulation, single-organism process, multiceller 
organismal process, binding, immune system process. It is worth noting that the VAP-1 protein is involved in the 13 most important biological processes. Using the online tool STRING to VAP-1 protein separation study found that: VAP-1 protein can participate in important functions such as complex inflammatory signaling pathways and receptor activities play an important role in biological processes. Using a single protein as a target, Dunkel et al (21) suggested that VAP-1 protein had a close relationship with other identification proteins (Fig. 4).

Amine oxidase, copper containing 3 (AOC3), is a major VAP-1 in endothelial cell surface adhesion molecule and its expression in the process of inflammation was obviously raised. AOC3 can increase the VAP-1 to adjust mediated neutrophil and other inflammatory cell adhesion, in order to migrate to the area of inflammation. After this progress endothelial cells can induce expression of intercellular adhesion molecule-1, IL-8. Kushimoto et al (22) found that blocking the expression of VAP-1 can significantly inhibit angiogenesis of inflammatory mediators, suggesting that VAP-1 can promote the occurrence and development of inflammation. Research shows that in many inflammatory diseases, such as psoriasis, chronic kidney disease, chronic liver disease, multiple sclerosis, VAP-1 content in peripheral blood increased significantly, which further prompts VAP-1 involvement in inflammation of the pathological process (23). In blood, VAP-1 protein is the carrier of vitamins and endotoxin playing an important role in vitamin metabolism. VAP-1 proteins may be combined with compound factor activation, enhance the regulation of information, regulating immune function, this function in different cells (initially monocytes and neutrophils) are found; the protein binds to the surface of the lymphocytes, specifically it binds to the membrane of the receptor on the lymphocytes (24). Once bound the protein complex is activated, and may initiate macrophages. As an autoimmune disease, SLE eye lesions may not only be the first manifestation, but may also be a prognostic as well as a predictive factor for the disease. Unlike connective tissue of the eyes, SLE can affect almost any part of the eye (eyelid, eye ball and dome conjunctiva), causing epithelial hyperplasia of fiber, resulting in conjunctivitis, and may release various inflammatory mediators and proteolytic enzymes, such as erosion on organization. Therefore, we hypothesized that the inflammatory response of VAP-1 may be related with caSLE, and directly or indirectly involved in inflammatory responses in the blood.

Proteomics offers unique advantages in the field of life science research, and provides a powerful tool in the study of many diseases such as SLE, attaining remarkable achievements. With the further development of proteomics technology, functional proteomics and biomarkers may be used to study SLE pathogenesis, targeted therapy and drug development, providing an important theoretical basis.

\section{Acknowledgements}

Not applicable.

\section{Funding}

No funding was received.

\section{Availability of data and materials}

The datasets used and/or analyzed during the present study are available from the corresponding author on reasonable request.

\section{Authors' contributions}

HL and HS were responsible for the conception and design of the study. JZ and PZ contributed to the collection and assembly of the data. JZ, MK and ND completed data analysis and interpretation, and HL and HS wrote the manuscript. All the authors read and approved the final manuscript.

\section{Ethics approval and consent to participate}

The study was approved by the Ethics Committee of the First Affiliated Hospital of Jiamusi University (Heilongjiang, China), and each patient signed an informed consent to participate in the study.

\section{Patient consent for publication}

Not applicable.

\section{Competing interests}

The authors declare that they have no competing interests.

\section{References}

1. Ferreira TA, de Andrade HM, de Pádua PM, Carvalho MD, Pires SD, Oliveira IH, Lima BS, Fialho Júnior LC, Cicarini WB, Chapeourouge DA, et al: Identification of potential biomarkers for systemic lupus erythematosus diagnosis using two-dimensional differential gel electrophoresis (2D-DIGE) and mass spectrometry. Autoimmunity 50: 247-256, 2017.

2. Hung WT, Chen YM, Lan JL, Chen HH, Chen YH, Chen DY, Hsieh CW and Wen MC: Antinucleosome antibodies as a potential biomarker for the evaluation of renal pathological activity in patients with proliferative lupus nephritis. Lupus 20 : 1404-1410, 2011.

3. Ravirajan CT, Rowse L, Mac Gowan JR and Isenberg DA: An analysis of clinical disease activity and nephritis-associated serum autoantibody profiles inpatients with systemic lupus erythematosus: A cross-sectional study. Rheumatology (Oxford) 40: 1405-1412, 2001.

4. Förger F, Matthias T, Oppermann M, Becker H and Helmke K: Clinical significance of anti-ds DNA antibody isotypes: Ig G/Ig M ratio of anti-ds DNA antibodies as a prognostic marker for lupus nephritis. Lupus 13: 36-44, 2004.

5. Trendelenburg M, Lopez-Trascasa M, Potlukova E, Moll S, Regenass S, Frémeaux-Bacchi V, Martinez-Ara J, Jancova E, Picazo ML, Honsova E, et al: High prevalence of anti-C1q antibodies in biopsy-proven active lupus nephritis. Nephrol Dial Transplant 21: 3115-3121, 2006.

6. Xiang YJ and Dai SM: Prevalence of rheumatic diseases and disability in China. Rheumatol Int 29: 481-490, 2009.

7. Murata M, Noda K, Fukuhara J, Kanda A, Kase S, Saito W, Ozawa Y, Mochizuki S, Kimura S, Mashima Y, et al: Soluble vascular adhesion protein-1 accumulates in proliferative diabetic retinopathy. Invest Ophthalmol Vis Sci 53: 4055-4062, 2012.

8. Solé M and Unzeta M: Vascular cell lines expressing SSAO/VAP-1: A new experimental tool to study its involvement in vascular diseases. Biol Cell 103: 543-557, 2011.

9. Feldman AS, Banyard J, Wu CL, McDougal WS and Zetter BR: Cystatin B as a tissue and urinary biomarker of bladder cancer recurrence and disease progression. Clin Cancer Res 15: 1024-1031, 2009.

10. Hotte NS and Deyholos MK: A flax fibre proteome: Identification of proteins enriched in bast fibres. BMC Plant Biol 8: 52, 2008. 
11. Liu J, Huang P, He Y, Hong WX, Ren X, Yang X, He Y, Wang W, Zhang R, Yang H, et al: Serum amyloid A and clusterin as potential predictive biomarkers for severe hand, foot and mouth disease by 2D-DIGE proteomics analysis. PLoS One 9: e108816, 2014.

12. Junker H, Venz S, Zimmermann U, Thiele A, Scharf C and Walther R: Stage-related alterations in renal cell carcinoma-comprehensive quantitative analysis by 2D-DIGE and protein network analysis. PLoS One 6: e21867, 2011.

13. Lappas M: Anti-inflammatory properties of sirtuin 6 in human umbilical vein endothelial cells. Mediators Inflamm 2012: 597514, 2012.

14. Yuan H, Yao YS, Chen GM, Sheng J, Xu L and Pan HF Decreased serum levels of T-cell immunoglobulin mucin-1 and T-cell immunoglobulin mucin-3 in systemic lupus erythematosus patients. J Biol Regul Homeost Agents 30: 123-129, 2016.

15. Penno MA, Klingler-Hoffmann M, Brazzatti JA, Boussioutas A, Putoczki T, Ernst M and Hoffmann P: 2D-DIGE analysis of sera from transgenic mouse models reveals novel candidate protein biomarkers for human gastric cancer. J Proteomics 77: 40, 2012.

16. Zhen Y, Xu N, Richardson B, Becklin R, Savage JR, Blake K and Peltier JM: Development of an LC-MALDI method for the analysis of protein complexes. J Am Soc Mass Spectrom 15: 803, 2004.

17. Deng L, Jia HL, Liu CW, Xu YF, Mao LJ, He CH, Yin GQ, Lin JH, Tao JP and Zhu L: Proteomic analysis of extremely severe hand, foot and mouth disease infected by enterovirus 71 BMC Infect Dis 13: 383, 2013.

18. Wong MY, Saad S, Pollock CA and Wong MG: Semicarbazidesensitive amine oxidase (SSAO) and kidney disease. Am J Physiol Renal Physiol 305: 1637-1644, 2013.
19. Yu SL, Kuan WP, Wong CK, Li EK and Tam LS: Immunopathological roles of cytokines, chemokines, signaling molecules, and pattern-recognition receptors in systemic lupus erythematosus. Clin Dev Immunol 2012: 715190, 2012.

20. Azkargorta M, Arizmendi JM, Elortza F, Alkorta N, Zubiaga AM and Fullaondo A: Differential proteome profiles in E2F2-deficient T lymphocytes. Proteomics 6 (Suppl 1): S42-S50, 2006.

21. Dunkel J, Aguilar-Pimentel JA,Ollert M,Fuchs H, Gailus-Durner V, de Angelis MH, Jalkanen S, Salmi M and Veres TZ: Endothelial amine oxidase AOC 3 transiently contributes to adaptive immune responses in the airways. Eur J Immunol 44: 3232-3239, 2014.

22. Kushimoto S, Shibata Y, Koido Y, Kawai M, Yokota H and Yamamoto Y: The clinical usefulness of procalcitonin measurement for assessing the severity of bacterial infection in critically ill patients requiring corticosteroid therapy. J Nippon Med Sch 74: 236-240, 2007.

23. Apostolidis SA, Lieberman LA, Kis-Toth K, Crispín JC and Tsokos GC: The dysregulation of cytokine networks in systemic lupus erythematosus. J Interferon Cytokine Res 31: 769-779, 2011.

24. Trivedi PJ, Tickle J, Vesterhus MN, Eddowes PJ, Bruns T, Vainio J, Parker R, Smith D, Liaskou E, Thorbjørnsen LW, et al: Vascular adhesion protein-1 is elevated in primary sclerosing cholangitis, is predictive of clinical outcome and facilitates recruitment of gut-tropic lymphocytes to liver in a substrate-dependent manner. Gut 67: 1135-1145, 2018 